\title{
Combinatorial Algorithm In Linear Model
}

\author{
Jana Ižvoltová ${ }^{1, *}$, and Peter Pisca ${ }^{1}$ \\ ${ }^{1}$ University of Žilina, Faculty of Civil Engineering, Department of Geodesy, Univerzitná 8215/1, \\ 01026 Žilina, Slovakia
}

\begin{abstract}
Gauss-jacobi combinatorial algorithm is an alternative approach to traditional iterative numerical methods, which is primary oriented for parameter estimation in nonlinear models. The combinatorial algorithm is often exploited for outlier diagnosis in nonlinear models, where the other parameter estimation methods lose their efficiency. The paper describes comparison of both of gauss-jacobi combinatorial and gauss-markov models executed on parameter estimation process of levelling network for the reason to find the efficiency of combinatorial algorithm in simply linear model.
\end{abstract}

\section{Introduction}

Gauss-Jacobi combinatorial algorithm is defined as numerical procedure for solving overdetermined problems, where more observations than unknown exist [1]. The advantage of the procedure is that the method does not require linearization, the need for iteration does not exist, the variance-covariance matrices of all parameters are considered and it can be exploited for outlier diagnosis.

The method was proposed by its author C. F. Gauss and was published after his death. Several years later, the method was developed by C. G. I. Jacobi who used the square of determinants as the weights in determining the unknown parameters from the arithmetic mean.

The principle of the method is based on obtaining the unknown value by minimizing the sum of square of errors of "pseudo-observations" formed from the combinatorial solutions.

\section{Principle of gauss-jacobi combinatorial algorithm}

Given $n$ algebraic observation equations with $u$ unknowns:

\footnotetext{
* Corresponding author: jana.izvoltova@,fstav.uniza.sk
} 


$$
\begin{aligned}
& a_{1} x+b_{1} y-y_{1}=0 \\
& a_{2} x+b_{2} y-y_{2}=0 \\
& a_{3} x+b_{3} y-y_{3}=0 .
\end{aligned}
$$

Solutions of the set of equations (1) obtained from each combinatorial pair of equations differ from the others due to the unavoidable random measuring errors. If the solutions from the pair of the combinatorial equations are $x_{1,2}, x_{2,3}, \ldots$ and $y_{1,2}, y_{2,3}, \ldots$, then the combined solutions are the sum of the weighted arithmetic mean:

$$
\begin{aligned}
& x=\frac{\pi_{1,2} x_{1,2}+\pi_{2,3} x_{2,3}+\ldots}{\pi_{1,2}+\pi_{2,3}+\ldots} \\
& y=\frac{\pi_{1,2} y_{1,2}+\pi_{2,3} y_{2,3}+\ldots}{\pi_{1,2}+\pi_{2,3}+\ldots},
\end{aligned}
$$

with $\pi_{1,2}, \pi_{2,3}, \ldots$ being the weights of the combinatorial solutions given by the square of the determinants as:

$$
\begin{aligned}
& \pi_{1,2}=\left(a_{1} b_{2}-a_{2} b_{1}\right)^{2} \\
& \pi_{2,3}=\left(a_{2} b_{3}-a_{3} b_{2}\right)^{2} .
\end{aligned}
$$

The specific characteristic of Gauss-Jacobi combinatorial algorithm is in identical results with those of least square solution, but only in its application in linear model. For nonlinear cases, the results of the combinatorial optimization may not coincide with those of least squares.

The principle of this method is in creating the minimal number of partial combinations of the model, which satisfies the combinatorial number:

$$
C_{k}=\left(\begin{array}{l}
n \\
u
\end{array}\right)=\frac{n !}{u !(n-u) !}
$$

The rank of the particular matrices of the particular $p$-models depends on the number of unknown parameters $u$. The solution of the combinatorial algorithm consists in estimating the introductory parameters $\boldsymbol{\beta}_{i}^{p}$ from the $i$-th equation of $p$-model as follows 


$$
\boldsymbol{\beta}_{i}^{p}=\left(\begin{array}{c}
\beta_{1}^{p} \\
\beta_{2}^{p} \\
\vdots \\
\beta_{u}^{p}
\end{array}\right)_{i}=\mathbf{A}_{i}^{p^{-1}} \mathbf{y}_{i}^{p}
$$

and in the defining the partial matrices:

$$
\mathbf{G}_{i}^{p}=\mathbf{A}_{i}^{p^{T}} \mathbf{P}_{i}^{p} \mathbf{A}_{i}^{p}
$$

which have to be positive definite and regular, because of their futher inversion. The unknown parameters of the mathematical model (5) are represented by the weighted averages, which are estimated from the equation:

$$
\overline{\boldsymbol{\beta}}=\left(\begin{array}{c}
\bar{\beta}_{1} \\
\bar{\beta}_{2} \\
\vdots \\
\bar{\beta}_{u}
\end{array}\right)=\left(\mathbf{G}_{i}^{p}+\mathbf{G}_{i+1}^{p}\right)^{-1}\left(\mathbf{G}_{i}^{p} \mid \mathbf{G}_{i+1}^{p}\right)\left(\frac{\boldsymbol{\beta}_{i}^{p}}{\boldsymbol{\beta}_{i+1}^{p}}\right)
$$

The proper identification of systematic influence in the model consists in comparison of a partial combinatorial positional norm estimated from the $i$-th model:

$$
K_{i}^{p}=\sqrt{x_{i}^{2}+y_{i}^{2}+z_{i}^{2}}
$$

with the median positional norm $K_{m e d}$ in case of robust estimation or with the "global" positional norm $K_{\bar{\beta}}$ estimated mean from the whole model, whereby the possible differences allocate the observation error.

The dimensions of the above known matrices depend on the rank of matrices, which represents the number of independent rows as follows:

$$
\operatorname{rank} \mathbf{A}_{i}^{p}=u, \operatorname{rank} \mathbf{D}(\mathbf{y})_{i}^{p}=u, \operatorname{rank}_{i}^{p}=u
$$

\section{Combinatorial algorithm in precise levelling}

Levelling network (Fig. 1) consists of two points with known heights $H_{1}$ and $H_{6}$ and four points with unknown heights $x_{1}, x_{2}, \ldots x_{4}$, which were joined by ten levelling routes represented by measuring values $h_{1}, h_{2}, \ldots h_{10}$. 


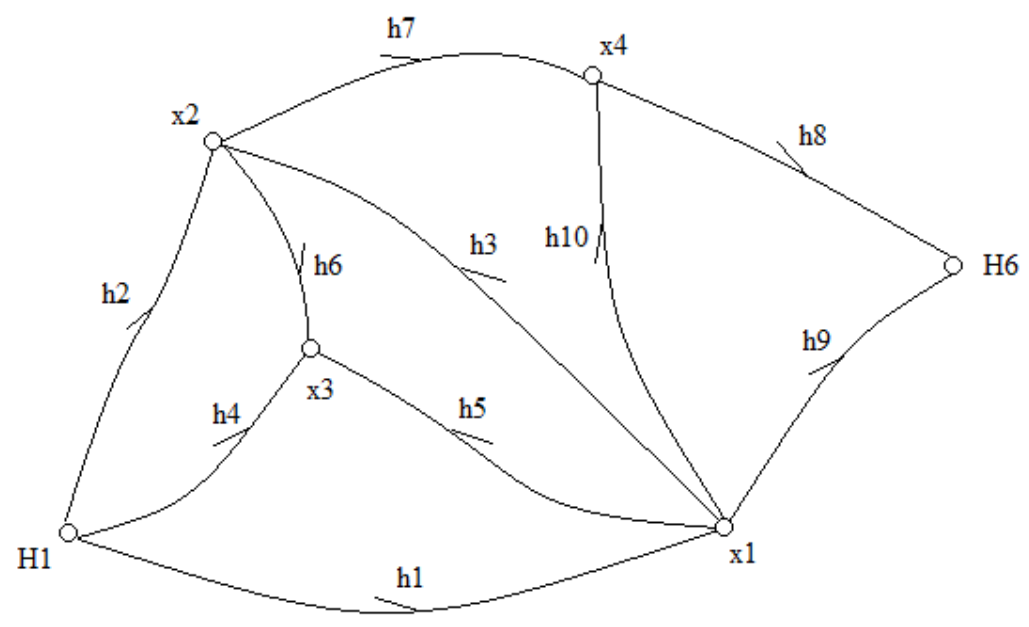

Fig. 1. Levelling network

Given six points with four of them being unknown, there exists fifteen combinations, to obtain the unknown heights:

$$
C_{k}=\left(\begin{array}{l}
6 \\
4
\end{array}\right)=\frac{6 !}{4 !(6-4) !}=\frac{6.5}{2}=15
$$

The construction of the partial models in precise levelling network depends on minimal number of levelling routes with the negotiable partial models, which use positive definite matrices. To keep this rule, we have chosen seven partial combinatorial models to estimate fours unknown heights. (Tab. 1). For the result comparison in every partial model, we also apply least square solution in Gauss-Markov model (Tab. 2).

Table 1. Estimations of unknown heights by Combinatorial algorithm

\begin{tabular}{|c|c|c|c|c|}
\hline Partial combinations & $\bar{\beta}_{1}=\bar{x}_{1}$ & $\bar{\beta}_{2}=\bar{x}_{2}$ & $\bar{\beta}_{3}=x_{3}$ & $\bar{\beta}_{4}=\bar{x}_{4}$ \\
\hline 1 & 344.3526 & 348.7500 & 352.5120 & 345.8722 \\
\hline 2 & 344.3525 & 348.7495 & 352.5121 & 345.8720 \\
\hline 3 & 344.3529 & 348.7499 & 352.5125 & 345.8721 \\
\hline 4 & 344.3529 & 348.7499 & 352.5123 & 345.8720 \\
\hline 5 & 344.3525 & 348.7494 & 352.5120 & 345.8719 \\
\hline 6 & 344.3527 & 348.7500 & 352.5122 & 345.8720 \\
\hline
\end{tabular}




\begin{tabular}{|c|l|l|l|l|}
\hline 7 & 344.3535 & 348.7504 & 352.5118 & 345.8726 \\
\hline Mean & 344.3528 & 348.7499 & 352.5121 & 345.8721 \\
\hline Median & 344.3527 & 348.7499 & 352.5121 & 345.8720 \\
\hline
\end{tabular}

Table 2. Estimations of unknown heights by Gauss-Markov model

\begin{tabular}{|c|c|c|c|c|}
\hline Partial combinations & $\bar{\beta}_{1}=\bar{x}_{1}$ & $\bar{\beta}_{2}=\bar{x}_{2}$ & $\bar{\beta}_{3}=x_{3}$ & $\bar{\beta}_{4}=\bar{x}_{4}$ \\
\hline 1 & 344.3525 & 348.7504 & 352.5118 & 345.8726 \\
\hline 2 & 344.3524 & 348.7493 & 352.5120 & 345.8722 \\
\hline 3 & 344.3524 & 348.7493 & 352.5119 & 345.8715 \\
\hline 4 & 344.3535 & 348.7504 & 352.5130 & 345.8726 \\
\hline 5 & 344.3522 & 348.7491 & 352.5118 & 345.8713 \\
\hline 6 & 344.3525 & 348.7495 & 352.5121 & 345.8722 \\
\hline 7 & 344.3535 & 348.7504 & 352.5118 & 345.8726 \\
\hline Mean & 344.3527 & 348.7498 & 352.5121 & 345.8721 \\
\hline Median & 344.3525 & 348.7495 & 352.5119 & 345.8722 \\
\hline
\end{tabular}

The deviations of estimated unknown parameters from the mean value (median) are shown in tab. 3. The insignificant differences between shadov and white part of tab. 3, allocate on the fact that Gauss-Jacobi algorithm seems to be more precise, which is confirmed by variance-covariance estimations. For example, the average value of standard deviation of Gauss-Jacobi algorithm is $\sigma_{G-J}=0.27 \mathrm{~mm}$ and Gauss-Markov model is $\sigma_{G-M}=0.49 \mathrm{~mm}$.

Table 3. Estimated parameters deviations in both of models

\begin{tabular}{|c|c|c|c|c|c|c|c|c|}
\hline \multirow{2}{*}{ Partial combinations } & \multicolumn{4}{|c|}{ Gauss-Jacobi } & \multicolumn{5}{c|}{ Gauss-Markov model } \\
\cline { 2 - 10 } & $\boldsymbol{\Delta}_{1}$ & $\boldsymbol{\Delta}_{2}$ & $\boldsymbol{\Delta}_{3}$ & $\boldsymbol{\Delta}_{4}$ & $\boldsymbol{\Delta}_{1}$ & $\boldsymbol{\Delta}_{2}$ & $\boldsymbol{\Delta}_{3}$ & $\boldsymbol{\Delta}_{4}$ \\
\hline 1 & 0.1 & 0.0 & 0.1 & -0.1 & 0.2 & -0.5 & 0.3 & -0.6 \\
\hline 2 & 0.2 & 0.4 & 0.0 & 0.1 & 0.3 & 0.6 & 0.1 & -0.2 \\
\hline 3 & -0.2 & 0.0 & -0.4 & 0.0 & 0.3 & 0.6 & 0.2 & 0.5 \\
\hline 4 & -0.2 & 0.0 & -0.2 & 0.0 & -0.8 & -0.5 & -0.9 & -0.6 \\
\hline 5 & 0.1 & 0.5 & 0.1 & 0.1 & 0.5 & 0.8 & 0.3 & 0.7 \\
\hline
\end{tabular}




\begin{tabular}{|c|c|c|c|c|c|c|c|c|}
\hline 6 & 0.0 & 0.0 & -0.1 & 0.0 & 0.2 & 0.4 & 0.0 & -0.2 \\
\hline 7 & -0.8 & -0.5 & 0.3 & -0.6 & -0.8 & -0.5 & 0.3 & -0.6 \\
\hline
\end{tabular}

\section{Conclusion}

The primary purpose of using Gauss-Jacobi combinatorial algorithm was to amend the endless iterative methods with the creating the minimal number of mathematical combinations of observed data. Formelly, authors of the combinatorial algorithm C. F. Gauss and C. G. I. Jacobi developed this method to simplify numerical solutions, but its application in the estimation process demonstrates the method's strength in its using in nonlinear models, especially in the proces of errors diagnostics referred to as outliers. The ambition of the paper was to show the possibility to apply the combinatorial algorithm also in the linear model, though the outlier identification is not so much effective. The results, published in the tab. 1. - tab. 3 allocate to more precise method in comparison with GaussMarkov model, which documents the values of standard deviations estimated in of GaussJacobi algorithm: $\sigma_{G-J}=0.27 \mathrm{~mm}$ and Gauss-Markov model: $\sigma_{G-M}=0.49 \mathrm{~mm}$. The difference of both of standard deviatons seems to be appreciable, especially concerning to the precise levelling observations.

This article is the result of the implementation of the project VEGA 1/0275/17 "Application of numerical methods to define the changes of geometrical track position", supported by the Scientific Grant Agency of the Ministry of Education, science, research and sport of the Slovak Republic and the Slovak Academy of Sciences.

This article is the result of the implementation of the project ITMS 26220220156 "broker centre of air transport for transfer of technology and knowledge into transport and transport infrastructure", supported by the research \& development operational programme funded by the ERDF

\section{References}

1. Awange, J. L., Grafarend, E. W.: Solving Algebraic Computational Problems in Geodesy and Geoinformatics (Springer Berlin, NewYork, 2005)

2. Grafarend, E. W.: Linear and Nonlinear Models. p.752 (Walter de Gruyter Berlin, New York, 2006)

3. Huber, P. J.: Robust Estimation of a Location Parameter, University of California, Berkeley

4. Rousseeuw, P. J. - Leroy, A. M.: Robust Regression and Outlier Detection. 333 p. (Wiley New Jersey, 2003)

5. Jurečková, J., Picek, J.: Robust Statistical methods with R. 197 p. (Chapman \& Hall/CRC Boca Raton 2006) 
6. Koch, K. R.: Parameter Estimation and Hypothesis Testing in Linear Models. Seit. 378 (Springer-Verlag, Berlin, 1988)

7. Gašinec, J., Gašincová, S., Sabová, J., Weiss, G.: Adjustment of position geodetic networks by robust estimations. In: 14th Conference of Association of Mining Surveyors and Geologists. Ostrava VŠB TU, 2007, 14 p., ISBN 9788024816548 